\title{
Interference Aware Cooperative Routing Algorithm for Wireless Ad Hoc Networks over Nakagami Fading and Lognormal Shadowing
}

\author{
Abdullah Waqas \\ Department of Electronics, Quaid-E-Azam University, Islamabad, Pakistan. \\ abdullah@ele.qau.edu.pk
}

\begin{abstract}
The quick deployment and flexible characteristics of wireless ad hoc networks make them suitable for many commercial and daily life applications. A most challenging task in these distributed networks is ensuring quality of service to end-to-end user in presence of wireless channel, random network topology, unpredictable traffic patterns, and shared resources. The performance of network is limit by the amount of interference in the network in case of simultaneous transmission over same frequency band. In this paper, we address interference issue in presence of nakagami fading and lognormal shadowing channel conditions and proposed an Interference Aware Cooperative Routing (IACR) approach to manage routing operations between nodes. The presented model reflects realistic scenarios for urban areas where fading and shadowing occurs due to tall buildings in the neighborhood of transmitters. The proposed solution establishes routes between source and destination by introducing cooperation among network nodes. Each node in the network selects path such that interference created by it towards other nodes is minimized taking into account that the Signal to Interference plus Noise Ratio (SINR) at destination does not decreases a threshold value. Hence, the presented approach reduces amount of overall interference in the network without degrading the quality of individual route. The simulation results show that the performance of proposed solution is better than conventional hop count and interference aware routing algorithms in terms of throughput and outage probability of the network.
\end{abstract}

Index Terms - Interference aware routing, cooperative routing, reactive protocols, ad hoc networks, nakagami fading, shadowing.

\section{INTRODUCTION}

Routing protocol design is an important parameter for network designer to establish communication among nodes in the network. The unavailability of central authority complicates route management in distributed scenarios [1]. In these networks, devices themselves manage network operations without in absence of central command. The independent decision making to acquire shared resources may instigate conflict of interest among nodes needs consideration while designing routing protocol. The routing can be cooperative to maximize overall network performance or can follow greedy approaches to maximize utility of each individual in the network $[2,3]$. Random channel conditions, shared resources, collision, congestion and high traffic load limits establishing high performance routes between the devices [4]. In mobile ad hoc networks, multiple sources can communicate with multiple destinations. The routes between source-destination pair are associated with one another which cannot be optimized individually. The selection of route between one source-destination pair influence communication between other nodes, therefore, there is a great need to search for a mechanism which jointly optimize the routes between the devices. In this paper, we propose Interference Aware Cooperative Routing (IACR) algorithm which elevates the performance of over-all network without degrading the communication among individual nodes. In the proposed algorithm each node selects the route which provides low interference to other nodes in the network which results in high network performance.

There are many routing protocols presented in literature to improve quality of communication in distributed scenarios. The simplest solution to establish routes between the nodes uses Minimum Hop Count (MHC) routing metric [5]. Although, the routes established by MHC routing metric minimizes time delay between the source and the destination but it is effected by interference in case of simultaneous transmission from other users transmitting over same frequency band. Another approach [6] estimates link quality to decide the best route towards destination form all possible routes. In this approach, number of retransmission between the source and destination is minimized to increase packet delivery ratio of the route. This approach does not grantee that the distance between source and destinations is minimized. Other approaches [7, 8] take into account the energy consumption of individual nodes to decide the best route form source to the destination. These approaches select the nodes which consumes less energy to deliver packets towards the destination. In [9] consider residual energy of nodes to select the routes to increase life time of the network. Some algorithms take interference as routing metric to improve performance of the network $[10,11]$. In [12] proposed a queuing model to select the route at which the congestion is minimized. Commonly, all above discussed algorithms uses greedy approaches in which each node maximizes its own 


\section{RESEARCH ARTICLE}

utility. Although the greedy approaches maximizes individual node utility but the performance of the over-all network may decreased. Some attention is focus by researchers to mitigate the selfish behavior and introduce cooperation among the nodes to improve the network performance [13, 14]. Many of these approaches tried to maximize network life time by introducing packet forwarding mechanism or removal of malicious nodes. A less attention focuses on the design of cooperative routing metrics. Moreover, mostly the presented algorithms are analyzed for simple channel which does not model realistic scenarios e effectively [15]. In [16] proposed interference aware routing metric to maximize network performance in terms of throughput for simple path loss model. The work does not present the effect on fading or shadowing on the algorithm which may decrease the performance of the algorithm. Similarly, [17] presents different routing strategies for path loss channel and does not considers effect of random channel on the performance of the algorithm.

In this paper, we present an interference aware cooperative routing algorithm to jointly improve performance of all the nodes in the network. In other words, the proposed solution maximizes overall performance of the network taking into account that the utility of individual node does not decrease a threshold level. The channel is model as nakagami-m fading and lognormal shadowing along with path loss. The model reflects the situations in urban areas, where high buildings create multi-paths and fading during communication. Interference is an important parameter to consider because if all the nodes transmit on same frequency band, the received power at the destination is affected by the amount of interference present in the environment [18]. In addition, thermal noise also degrades the performance of the link. The proposed solution chooses the nodes in the route which creates low interference to the other nodes keeping in view that the data is successfully received at the destination. This cooperative behavior de-creases overall created interference in the network which consequently improves the performance of overall network.

The paper is organized as follows. Section II discusses literature survey. The section briefly discuss the algorithm and drawbacks of the previously propose schemes. In section III we discuss the system model used to check the performance of the proposed algorithm. Section IV shows the presented algorithm in detail. Section V shows simulation results which shows that the proposed solution performs better than previously proposed algorithms. In section VI we conclude the discussion.

\section{RELATED WORK}

In the field of distributed network design algorithms, many attempts are made for design of routing protocols for ad hoc networks $[19,20,21,22]$. Researchers explore diverse disciplines such as dynamic programming [23], algorithm design [24], graph theoretical algorithms [4] and game theory [25] to propose an efficient routing algorithm for regular as well as random network topologies. The selection of route depends on geographical position of source and the destination node, network topology, and channel conditions. In addition, limited bandwidth, heavy traffic load, and simultaneous transmissions from multiple users complicate the route establishment and management operation.

There are many routing metric presented by researchers which incorporate some of the above mentioned network parameters while establishing routes ranging from simple hop count routing metrics to comparatively complex energy aware and link-topology based routing metrics. X. Zhang el al in [26] presented a hop count routing protocol that have trade-off with transmission power of the nodes. The presented work incorporate asymmetric links and heterogeneous transmission power while establishing routes between nodes. The presented approach utilizes high power nodes in the network to reduce number of hops in the route. The presented results shows that the proposed Efficient Hop Count Routing (EHCR) approach improves the packet delivery ratio and normalized traffic overhead of the network for simple path-loss channel conditions, as compared to Ad hoc On-demand Distance Vector (AODV) and Dynamic Source Routing (DSR) routing protocols. In [27] a load aware routing metric is pro-posed which used Medium Access Control (MAC) layer channel contention information, number of packets in interference queue, and hop count to estimate the quality of link. In [28] proposed another link quality aware routing protocol using topological information to decide the next hop in the route. The metric used by this algorithm takes into account the weighted sum of remaining energy, link quality and progress to compute the next hop from neighbors. The link quality is determined by physical layer parameters. The parameter progress includes the effects of change in quality of link in case of mobile nodes and random channel conditions. In [29] presented interference aware routing protocols named iAware to select low interference path from source to the destination in multi-radio wireless mesh networks. The presented approach captures the effect of link quality, data transmission rate as well as inter-flow and intra-flow interference to establish communication links between the nodes.

In [30] proposed an interference aware routing metric for Optimized Link State Routing (OLSR) protocol. Taking the advantage of heterogeneous power capabilities of devices, the transmission range is divided into patches to achieve precise calculation of interference. Each patch is assigned a weight which depends on the distance between the source and interfering node. The interference at receiver is weighted sum of all the interfering nodes. The results show that the proposed solution provides better packet delivery and low routing overhead as compared to traditional OLSR routing 


\section{RESEARCH ARTICLE}

protocol.

The interference aware energy efficient routing (IAEE) algorithm proposed in [18] selects the routes which provide low interference to the data packet results in better routes as compared to $\mathrm{MHC}$ routing technique. In this technique source node selects the next hop which has lowest interference from all of its neighbors. Reducing the interference increases SINR at that link which results in high through-put and low energy consumption at that link. Each subsequent node follows the similar procedure and the algorithm returns minimum interference route between source and the destination. Simulation results show that the through-put of IAEE is higher as compared to hop count and energy aware routing protocols.

The routing metric propose in [9] takes into account the residual energy of the nodes, interference and link capacity to construct routes between the source and the destination. The results are analyzed for random channel conditions, where fading and shadowing affects the communication. The presented results show that the performance of proposed solution is better than traditional algorithms.

Most of the routing approaches discussed above do not incorporate random channel conditions while establishing routes between the nodes in the network. In this paper, we utilize cooperation among nodes to establish routes taking into account the random channel conditions such as path loss, fading, and shadowing. In the next section, we discuss system model used to study the performance of the network.

\section{SYSTEM MODEL}

Wireless ad hoc networks consist of wireless devices randomly distributed in the network area. The location of each node is independent of other nodes. A device can be placed at any location in the deployment area with equal probability. In other words, the spatial distribution of the nodes is uniform. We have $\mathrm{N}$ nodes uniformly distributed in the network area A. The network area can have any shape (circular, hexagonal, square, etc.) but we consider square deployment region to simplify the calculations. The assumption did not affect the algorithm and the results can be easily modified for other shape of area.

Each node in the network is equipped with an omnidirectional antenna transmit in circular region with node at center of the circle. The radius of transmission circle $r_{t}$ depends on the transmission power Pt of the node. We assume that all the nodes transmit with same transmission power on same frequency band. Moreover, the energy reservoirs of the nodes are limited. Each node in the network can configure itself as transmitter, receiver and/or relay node to accomplish the task of cooperative network.

In relay mode, the node forwards the traffic of other nodes alongside its own data traffic.

When a node desires a communication link with a destination which is outside its communication radius, it uses its neighbor as relay node to establish multi-hop route towards the destination. We assume that there is no selfish/malicious node in the network and all the nodes work in cooperative environment. The power reached at the receiver follow decaying pattern according to path-loss law which is,

$$
P_{r}=\frac{P_{t}}{d^{\alpha}}
$$

Where $\alpha$ is path-loss exponent whose value depends on the channel conditions.

Moreover, the performance of wireless system is limited by fading and shadowing effects. The fading occurs due to multipath in the channel which is modeled by Nakagami-m distributed RV denoted by $\mathrm{G}$ whose probability density function is,

$$
f_{G}(g ; m ; \sigma)=\frac{2 m^{m}}{\Gamma(m) \sigma^{m}} \mathrm{~g}^{2 \mathrm{~m}-1} \exp \left(-\frac{\mathrm{m}}{\sigma} \mathrm{g}^{2}\right)
$$

The large scale fading due to shadowing in the channel is modeled as lognormal distributed RV denoted by $\mathrm{S}$ given as,

$$
f_{S}(s)=\frac{1}{s \sigma \sqrt{2 \pi}} \exp \left(-\frac{(\ln \mathrm{s})^{2}}{2 \sigma^{2}}\right)
$$

In this case, Eq. (1) modifies as,

$$
P_{r}=\frac{P_{t} G S}{d^{\alpha}}
$$

The interference on the receiver is the sum of received power form all the nodes except source node which can be written as,

$$
I_{r}=\sum_{k=1, k \neq s}^{N} \mathrm{P}_{\mathrm{k}}
$$

The Signal to Interference plus Noise Ratio (SINR) can be written as,

$$
\beta_{n}=\frac{P_{s}}{I_{r}+\sigma^{\alpha}}
$$

Where $\sigma^{2}$ is the power of additive white noise. The power of noise is very low as compared to interference power so the above equation can be modified as, 


\section{RESEARCH ARTICLE}

$$
\beta=\frac{P_{s}}{I_{r}}
$$

A packet is successfully received at the destination node if $\beta$ at each link in case of multihop route is greater than a threshold $\beta_{\text {th }}$. The selection of threshold depends on the decoding algorithm.

\section{INTERFERENCE AWARE COOPERATIVE ROUTING}

In previous section we shows that the MHC and IAR routing protocols are not always beneficial for network in case of random channel conditions. The main factor which limits the performance of network is interference created by nodes. MHC routing metric makes routes on the base of hop count between the nodes and does not take into account the interference created on the route. The established route has minimum number of hops towards the destination which reduces time delay during communication but in many scenarios the interference on the routes created by MHC is high which decreases throughput of the network. The interference power increases when nodes in the network increase. This high interference reduces SINR at the route as well as individual links which reduces outage probability of the established communication path. In our previous work [18], we proposed interference aware energy efficient routing algorithm to improve the performance of the network. The proposed solution also provides high throughput as compared to $\mathrm{MHC}$ routing algorithm. In this paper, we propose new routing metric in which nodes select routes cooperatively and maximize performance of overall network.

When source node wants to communicate with a destination node, it initiates a route discovery process and locates the destination within network area. In multi-hop networks, multiple paths towards the destination can exist. The selection of next hop for a multi-hop route is based on the metric value. IACR algorithm uses interference as routing metric which need to minimize. The source node calculates the interference value created to other node when it transmits to its $j$-th neighbor. It also collects the information about the value of interference received at the $\mathrm{j}$-th node. The created and received interference values are summed to get the value of metric. The process is repeated for all neighbors and the node which has minimum metric value is selected as next hop towards the destination. The accommodation of created interference in calculation of routing metric introduces cooperative behavior in communication. In this case, a link which have high SINR value may not be used for established route because if it decreases the SINR at other nodes by providing higher interference to those nodes.

In the presented approach, the node select the next hop among its neighbors such that the interference created in the network is minimized keeping in view that the SINR at the link is greater than threshold SINR. Hence, the routing algorithm minimizes the created interference in the network without decrease in quality of the communication. The metric value used to select next hop is,

$$
M=\mathrm{I}_{\mathrm{c}}+\mathrm{I}_{\mathrm{r}}
$$

Where $I_{c}$ represent the interference created by transmitter and $\mathrm{I}_{\mathrm{r}}$ is the interference received at the destination node. The used metric jointly reduces the created and received interference.

\subsection{Information Collection Phase}

In first phase of algorithm, the source node sends an information collection packet to its neighbors which contain the address of the source node. Each neighbor when receives this packet sends a reply back to the source node which contains the value of interference received by him from the other nodes as well as the power received from the source node. The source node manages an information table in which it contains the interference created by him to other nodes along with the address of the node. When the information from all of its neighbors is successful, it invokes the route establishment phase. The information contents are directly extracted from route request packet and there is no need for an extra packet. The algorithm for information collection phase is given in table 1 .

Table 1: Information Collection Phase

\begin{tabular}{|l|}
\hline For each node (t) \\
\hline While transmitter \\
ICReq= information collection Request packet \\
$\mathrm{n}=$ array of address of neighbors \\
$\mathrm{i}=1$ \\
While not empty $\mathrm{n}$ \\
ICReq[destination.ID]=n(i).ID \\
ICReq[transmitter.ID]=t.ID \\
ICReq[transmitPower]=t.Power \\
transmit ICReq to neighbors \\
remove n(i) from neighbor list \\
endwhile \\
endwhile \\
While receiver \\
if PacketType=ICReq \\
if ICReq[destination.ID]=node.ID \\
ICRep[receivePower]=calculated receive \\
power $\mathrm{P}_{\mathrm{r}}$ \\
ICRep[receiveInterference]=calculated \\
interference $\mathrm{I}_{\mathrm{r}}$ from neighbors \\
ICRep[destination.ID]=[node.ID] \\
send ICRep packet to node t \\
else ICReq[destination.ID] =node.ID \\
ICRep[receivePower]=calculated receive \\
\hline
\end{tabular}




\section{RESEARCH ARTICLE}

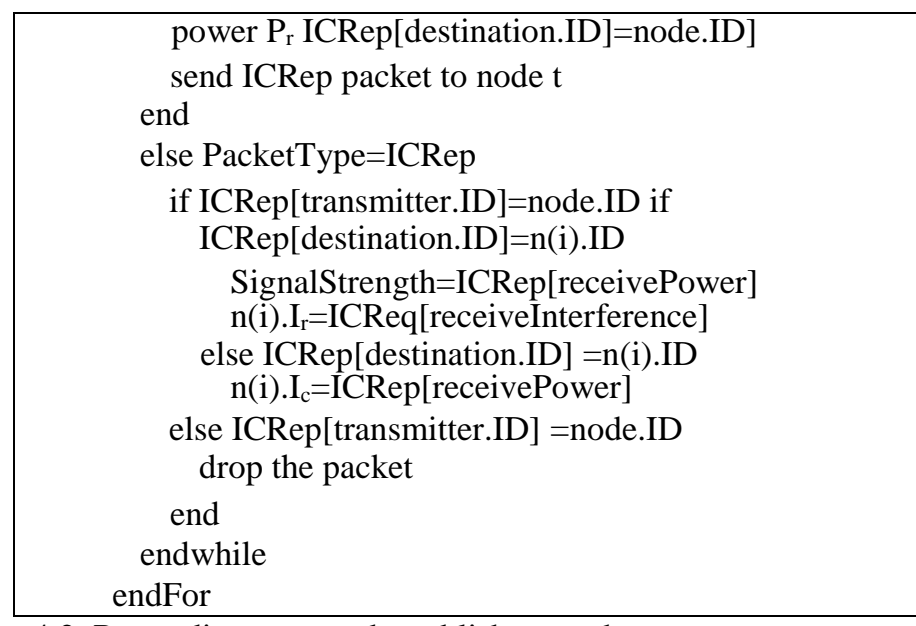

4.2. Route discovery and establishment phase

When a source node wants to communicate with a destination node, it initiates a route discovery process. In this process, the source node locates the destination within network area. The search is initiated with the transmission of route request (RREQ) packet towards the neighbors. The format of standard AODV route request packet is shown in table 2. The conventional routing protocol uses hop count as routing metric. In this work, we use IACR metric which improves the performance of the network. The metric value is computed by Eq. (8). The node which has least value of metric is used to relay RREQ packet towards the destination so that the cost of communication is minimized. When an intermediate node in the route receives the packet, it saves the identity of the node from which it receives the RREQ to keep track of reverse path. When the RREQ packet reaches the destination node, it acknowledge with RREP packet on reverse path. Each relay node when receives RREP packet, it updates its routing table and forwards the packet to the node on reverse path. When RREP reaches the source, a route is established between source and destination. The established route has minimum value of metric. The algorithm for this phase is shown in table 3.

\subsection{Throughput Analysis}

Throughput of the networks an important parameter while designing routing protocol. Throughput of the route depends on the quality of worst link in the route. The throughput of a route depends on the channel conditions, congestion, and interference experienced by the nodes in the route.

Table 2: Format of RREQ Packet

\begin{tabular}{|l|l|l|l|}
\hline Type & Flags & Reserved & Hop count \\
\hline \multicolumn{3}{|c|}{ RREQ ID } \\
\hline
\end{tabular}

\begin{tabular}{|c|}
\hline Destination IP Address \\
\hline Destination Sequence Number \\
\hline Originator IP Address \\
\hline Originator Sequence Number \\
\hline
\end{tabular}

Table 3: Route discovery and establishment phase

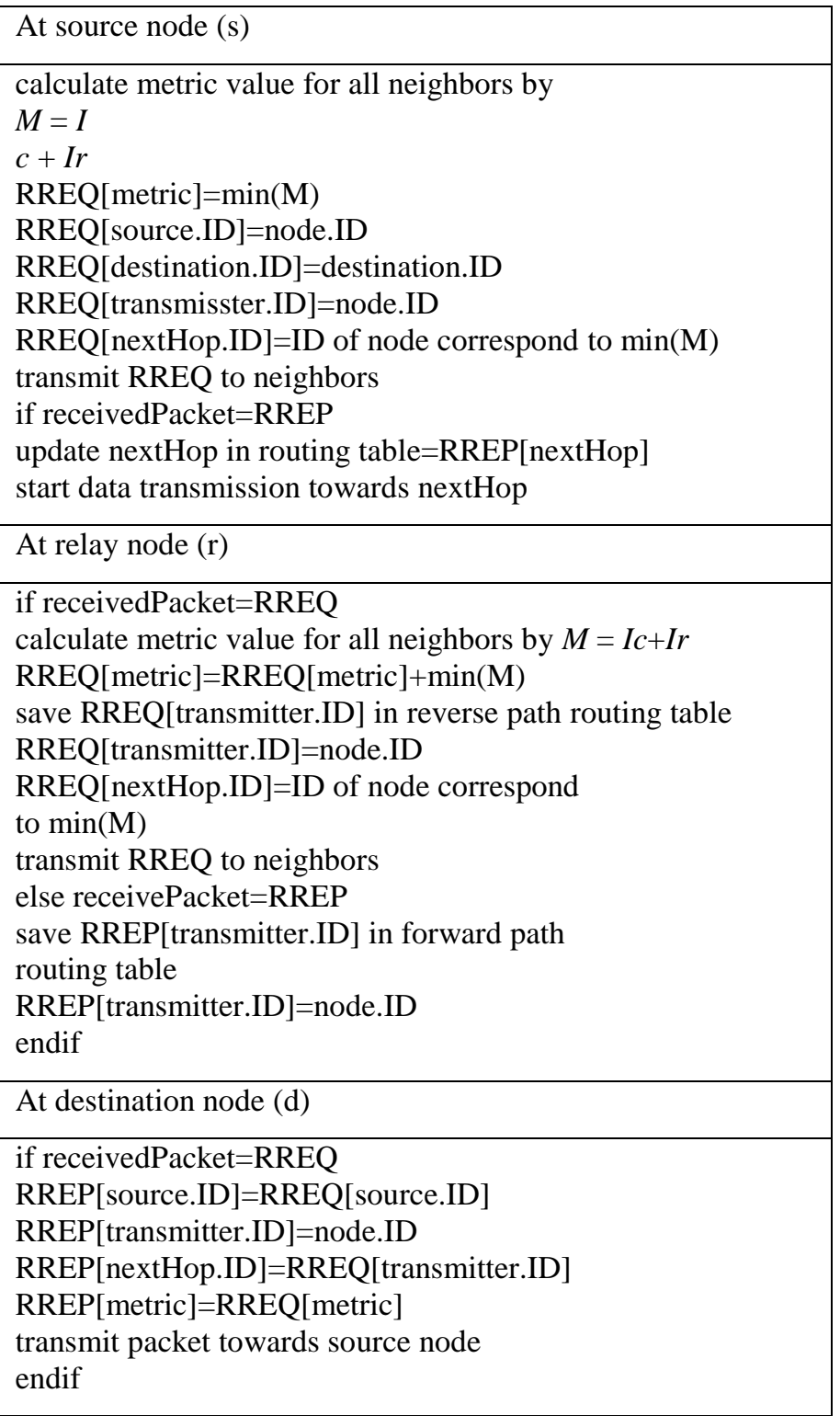

The worse channel condition results in lower throughput. The throughput is defined as the ratio of number of packets successfully received at the receiver to the total number of packets transmitted by the source node. Suppose $\tau$ represents throughput of a links then, 


\section{RESEARCH ARTICLE}

$$
\tau=\frac{n_{s}}{T}
$$

Where $n_{s}$ represents the number of packets successfully received at the receiver and $\mathrm{T}$ represents the total number of packets transmitted towards the destination. A packet is successfully receive if the SINR at the receiver is grater that a threshold. Suppose $\gamma_{\text {th }}$ represents the threshold value required to successfully decode the packet and $\gamma$ is the received SINR at the receiver then,

$$
n_{s}= \begin{cases}1 & \gamma \geq \gamma_{\mathrm{th}} \\ 0 & \gamma<\gamma_{\mathrm{th}}\end{cases}
$$

Above equation shows that the probability that the packet is successfully received at the receiver depends on the SINR at that link which depends on the channel condition and interference at the link. The IACR algorithm selects the nodes which creates low interference to the network which results in high SINR or equivalently high throughput routes.

\subsection{Outage Probability}

In multi-hop wireless systems, packet travels multiple hops before reaching to the destination. If relaying is analog, intermediate nodes amplify and forward the data packet received from the source node. The end-to-end outage probability of the route is defined as the probability that the route $\operatorname{SINR}\left(\gamma_{\mathrm{r}}\right)$ is below some threshold value $\left(\gamma_{\mathrm{th}}\right)$ which can be written as,

$$
P_{\text {out }}=\operatorname{Pr}\left\{\gamma_{r} \leq \gamma_{t h}\right\}
$$

Where $\gamma_{r}$ is defined in [31] as,

$$
\gamma_{r}=\left[\sum_{n=1}^{k} \frac{1}{\gamma_{n}}\right]^{-1}
$$

Where $\gamma_{\mathrm{n}}$ represents the SINR of the $\mathrm{n}$-th link in the route and $\mathrm{k}$ represents total number of links in the route. The above equation shows that the outage probability depends on the quality of individual link as well as the number of hops in the route.

\section{SIMULATION RESULTS}

\subsection{Simulation setup}

The network consists of $N$ wireless devices uniformly distributed in a square area of A $\left(\mathrm{m}^{2}\right)$. The node are configured to operate in ad hoc mode of operation, therefore, each node can act as source, destination or relay node. Each node is equipped with an omni-directional antenna which can transmit in a communication circle of radius $r_{t}$. The network establishment time is taken as 3 seconds, in which, the node discover their neighbors by transmitting "HELLO" packets in their communication circle. The node transmit "HELLO" packet after every 0.2 seconds to keep updated snapshot of their neighbors.

When source node desires communication link, it initiates route discovery algorithm. We used conventional ERS as destination discovery algorithm in our simulation setup. In presented setup, multiple nodes can transmit their data simultaneously creating interference for each other. We assume that a source node can start request a communication link with a destination at any time after establishment of the network. The source node transmits message packet of 512B after every 0.1 second after establishment of route. The SINR at receiver is affected by fading and shadowing effects present in wireless channel. The received signal power at the destination is calculated form Eq. (1). The packet is successfully received at the destination if SINR at receiver is greater than threshold value of SINR which depends on underlying modulation and coding technique. We used simple BPSK modulation in our simulation setup to highlight the effect of routing algorithm on performance of network.

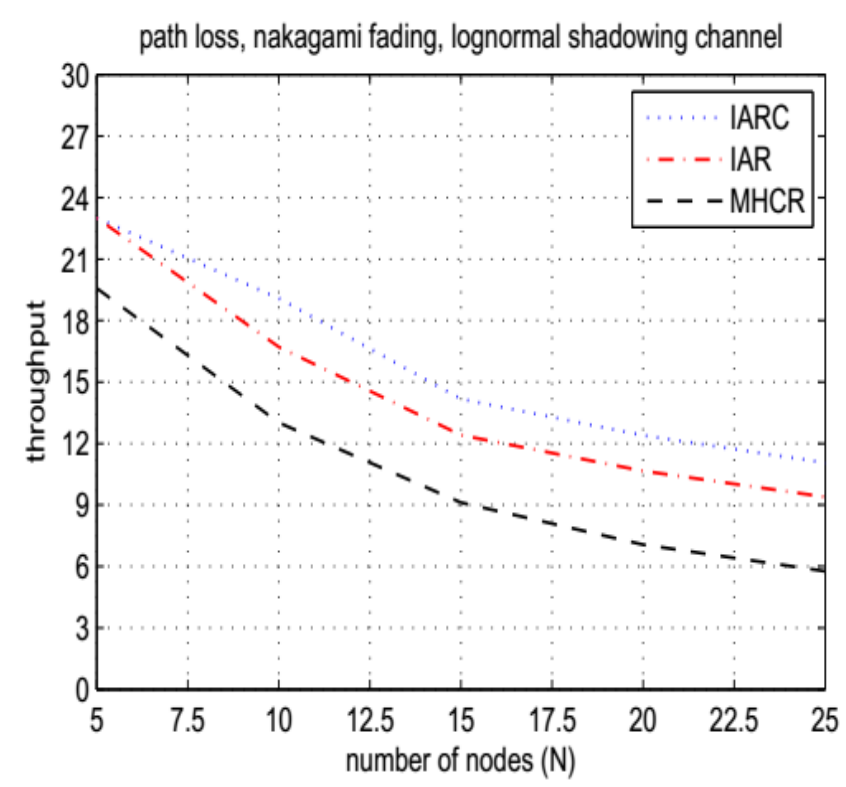

Figure 1 Throughput of different routing metrics over path loss, fading and shadowing channel

\subsection{Results and discussions}

Figures 1 shows the throughput $(\mathrm{KB} / \mathrm{Sec})$ of the network for various routing metrics and different channel conditions. The graph shows the scenario of urban environment where path loss, fading and shadowing effects the communication. The graph shows that the throughput of the network decreases with increase in number of nodes in the network. The 


\section{RESEARCH ARTICLE}

graph shows that IARC performs better than MHC and IAR routing metrics. The IARC metric uses both the created and received interference to compute route between nodes which results in low interference in the network which results in high network throughput. The plot shows that the result of $\mathrm{MHC}$ is worst from all of the techniques because it does not accommodate the characteristics of interference while deciding to establish routes between the source and the destination.

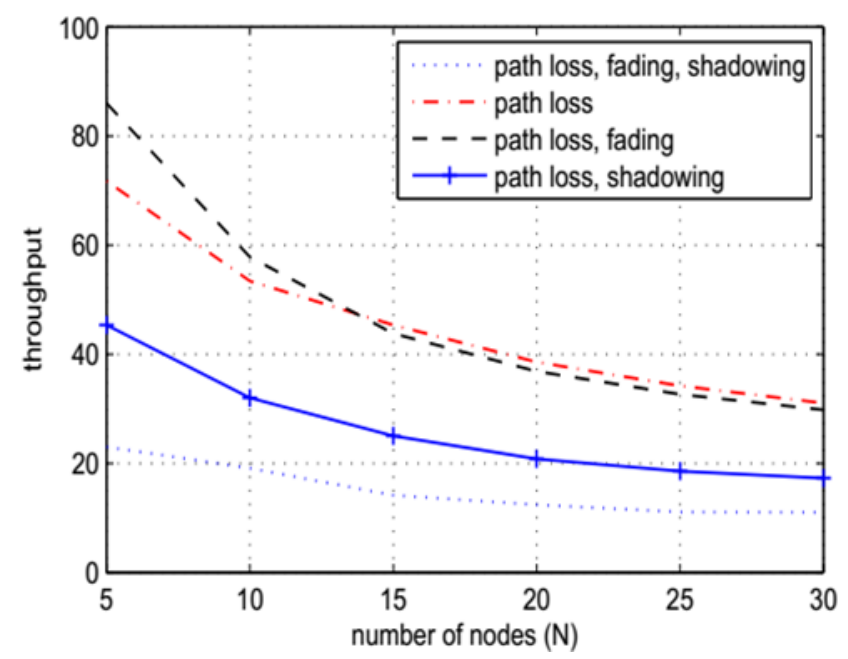

Figure 2 Throughput of IARC for Various Channel Conditions

Figure 2 shows the analysis of IARC for various channel conditions. The graph shows that the throughput of IARC decreases with increase in number of nodes in the network. The graph shows that the throughput of the channel when both fading and path loss are present in the network is almost same as compared to simple path loss channel. The trend shows that fading is a minor component to degrade the throughput of the network. In the channel conditions, where shadowing is present alongside path loss, the throughput of the network is degraded. The network performance is worst if path loss, fading and shadowing effects the communication.

Figure 3 shows the comparison of outage probability of different routing schemes. The results show that the outage probability increases with respect to SIR threshold. Increasing SIR threshold requires comparatively high received power to decode the packet successfully which results and increase in outage probability of the route. The graph shows that the presented IACR approach performs better than previous schemes. The performance of IARC is almost equal to IAR algorithm when threshold SIR is high. At high threshold values, the node requires high transmission power to achieve target threshold value which results in increase interference and consequently increase in outage.

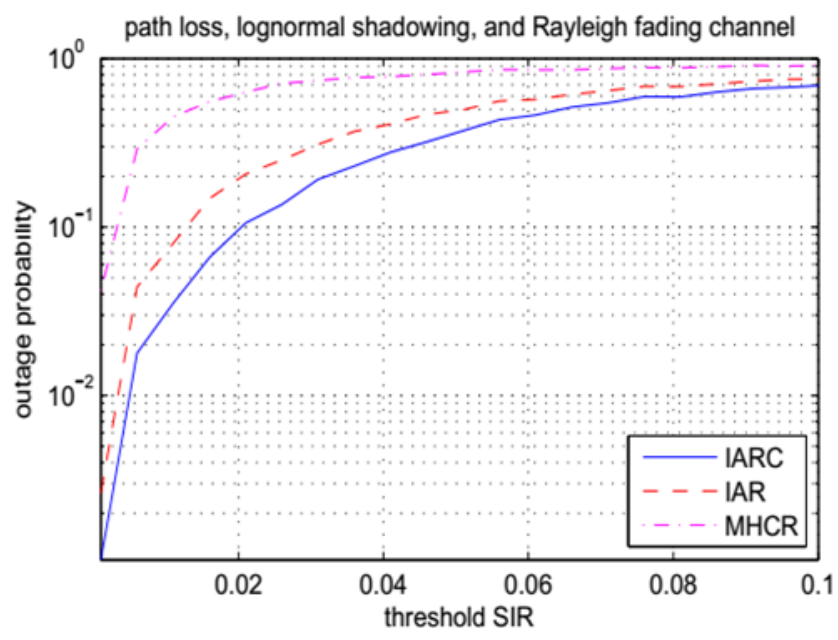

Figure 3 Outage Probability of Presented Routing Schemes for Various Channel Conditions

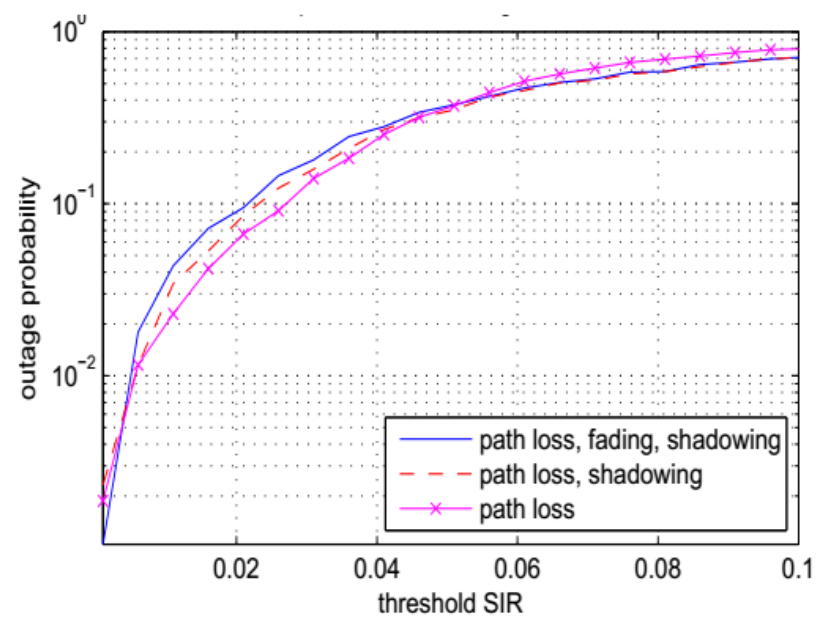

Figure 4 Outage Probability of IARC for Different Channel Conditions

Figure 4 shows the outage probability of IACR for various channel conditions. The graph shows that IARC performs better in path loss environment if SIR threshold is relatively low. In case of high SIR threshold, the IACR performance is almost independent from channel conditions. When SIR threshold value is high, the nodes require high transmission power to successfully transfer data towards the destination which results in increase in outage probability.

\section{CONCLUSIONS}

In this paper, we shows that the cooperation among network nodes is an important parameter which need consideration when deciding routes between the nodes in unpredictable channel conditions. Most of the conventional 


\section{RESEARCH ARTICLE}

routing algorithms do not consider interference between the network nodes while taking routing decisions. We show that introducing cooperation among the nodes significantly improves the performance of overall network. We show that the presented cooperative interference aware routing metric improves performance of the distributed networks in random channel conditions.

\section{REFERENCES}

[1] Zafar, Saima, Hina Tariq, and Kanza Manzoor, Throughput and Delay Analysis of AODV, DSDV and DSR Routing Protocols in Mobile Ad Hoc Networks, International journal of computer networks and applications 2(3)(2016), pp. 25-31.

[2] H. Chen, W. Lou, Gar: Group aware cooperative routing protocol for resource-constraint opportunistic networks, Computer Communications

[3] Naidu, Santosh, Mitigation of Energy Depletion in Wireless Ad-hoc Sensor Networks through Path Optimization, International journal of computer networks and applications 1(2)(2015), pp. 01-11.

[4] N. C. S. Iyengar, S. Saha, et al., An efficient interference aware partially overlapping channel assignment and routing in wireless mesh networks, International Journal of Communication Networks and Information Security (IJCNIS) 6 (1).

[5] R. Draves, J. Padhye, B. Zill, Comparison of routing metrics for static multi-hop wireless networks, ACM SIGCOMM Computer Communication Review 34 (4) (2004) 133-144.

[6] D. D. Mai, A. T. Tran, M. K. Kim, Measuring link quality based on etx metric in multi-hop wireless networks

[7] K. Parkavi, P. Vivekanandan, Energy aware secure routing protocol for mobile adhoc networks, ENERGY 2 (5).

[8] V. Mor, H. Kumar, Energy efficient wireless mobile networks: A review, in: Optimization, Reliabilty, and Information Technology (ICROIT), 2014 International Conference on, IEEE, 2014, pp.281-285.

[9] Z. Huang, R. Yamamoto, Y. Tanaka, A multipath energyefficient probability routing protocol in ad hoc networks, in: Advanced Communication Technology (ICACT), 2014 16th International Conference on, IEEE, 2014, pp. 244-250.

[10] J. Lu, X. Wang, L. Zhang, Signal power random fading based interference-aware routing for wireless sensor networks, Wireless Networks(2014)1-13.

[11] S. Touati, H. Boujemaa, N. Abed, Outage probability analysis of optimal and suboptimal interference aware routing protocols for multihop underlay cognitive radio networks, Transactions on Emerging Telecommunications Technologies.

[12] Y. Dong, P. Du, Cross-layer design of $2 \mathrm{~d}$ queuing model for multi-hop wireless networks, Wireless Personal Communications (2014) 1-18.

[13] J. Duan, D. Yang, H. Zhu, S. Zhang, J. Zhao, Tsrf: A trustaware secure routing framework in wireless sensor networks, International Journal of Distributed Sensor Networks 2014.

[14] S. SENTHILKUMAR, J. WILLIAM, A survey on reputation based selfish node detection techniques in mobile ad hoc network., Journal of Theoretical \& Applied Information Technology 60 (2).

[15] M. A. Razzaque, M. H. U. Ahmed, C. S. Hong, S. Lee, Qosaware distributed adaptive cooperative routing in wireless sensor networks, Ad Hoc Networks.

[16] H. Mahmood, C. Comaniciu, Interference aware cooperative routing for wireless ad hoc networks, Ad Hoc Networks 7 (1) (2009) 248-263.

[17] B. Gui, L. Dai, L. J. Cimini, Routing strategies in multihop cooperative networks, Wireless Communications, IEEE Transactions on 8 (2) (2009) 843-855.

[18] A. Waqas, H. Mahmood, Interference aware adaptive routing for cdma based mobile ad hoc networks, in: Intelligent Systems
Modelling \& Simulation (ISMS), 2013 4th International Conference on, IEEE, 2013, pp. 492-496.

[19] S. Mahajan, Performance comparison of dynamic source routing under varying traffic patterns, Networking and Communication Engineering 6 (2).

[20] N. Kishore, S. Singh, R. Dhir, Energy based evaluation of routing protocol for manets.

[21] B. Sivakumar, N. Bhalaji, D. Sivakumar, A survey on investigating the need for intelligent power-aware load balanced routing protocols for handling critical links in manets, The Scientific World Journal 2014.

[22] S. Goswami, S. Joardar, C. B. Das, Reactive and proactive routing protocols performance metric comparison in mobile ad hoc networks ns 2, memory 3 (1).

[23] Y. Qin, Q. Zhang, S. Wang, J. Li, Selective quality routing algorithm based on dynamic programming in wireless mesh networks, in: Proceedings of the 2012 International Conference on Cybernetics and Informatics, Springer, 2014, pp. 1997-2005.

[24] G. Kumar, N. Mishra, A. P. Singh, O. P. Kushwaha, A novel (vol-routing) page rank based on visit of links routing algorithm method in ad-hoc wireless networks, in: Issues and Challenges in Intelligent Computing Techniques (ICICT), 2014 International Conference on, IEEE, 2014, pp. 435-438.

[25] A. H. Dehwah, H. Tembine, C. Claudel, a decentralized routing scheme based on a zero-sum game to optimize energy in solar powered sensor networks, in: Proceedings of the 13th international symposium on Information processing in sensor networks, IEEE Press, 2014, pp. 285-286.

[26] X. Zhang, Z.-H. Qian, Y.-Q. Guo, X. Wang, An efficient hop count routing protocol for wireless ad hoc networks, International Journal of Automation and Computing 11 (1) (2014) 9399.

[27] Y. Li, H. Man, Three load metrics for routing in ad hoc networks, in: Vehicular Technology Conference, 2004. VTC2004Fall. 2004 IEEE 60th, Vol. 4, IEEE, 2004, pp. 2764-2768.

[28] Z. Zhao, D. Ros’ario, T. Braun, E. Cerqueira, H. Xu, L. Huang, Topology and link quality-aware geographical opportunistic routing in wireless ad-hoc networks, in: Wireless Communications and Mobile Computing Conference (IWCMC), 2013 9th International, IEEE, 2013, pp. 1522-1527.

[29] A. P. Subramanian, M. M. Buddhikot, S. Miller, Interference aware routing in multi-radio wireless mesh networks, in: Wireless Mesh Networks, 2006. WiMesh 2006. 2nd IEEE Workshop on, IEEE, 2006, pp. 55-63.

[30] V. L. SV, E. Prakash, P. Scholar, A novel interference aware optimized link state routing protocol for power heterogeneous manets.

[31] M. O. Hasna, M.-S. Alouini, Outage probability of multihop transmission over nakagami fading channels, Communications Letters, IEEE 7 (5) (2003) 216-218.

Author

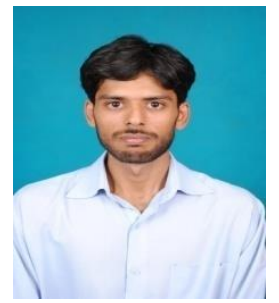

Abdullah Waqas received my M.Phil degree from Quaid-I-Azam University, Islamabad, Pakistan in 2010. Currently, he is Ph.D student in Department of Electronics. He worked as faculty member in Department of Electronics, Quaid-IAzam University, Islamabad from 2010 to 2012. His research interests include wireless communication, network protocols, game theory, and ad hoc and sensor networks. 